\title{
Justice Towards Animals Demands Veganism
}

\author{
Gary Steiner
}

Adapted from Gary Steiner, Animals and the Limits of Postmodernism: A Vegan Manifesto (Columbia University Press, forthcoming 2012).

In November 2009, the Sunday immediately prior to the Thanksgiving holiday traditionally celebrated in the United States, I published an opinion piece in the New York Times entitled 'Animal, Vegetable, Miserable'. 'Animal, Vegetable, Miserable,' New York Times, 22 November, 2009, 'Week in Review' 12. The traditional Thanksgiving meal is centred around turkey, and my aim in writing the opinion piece was to challenge the supposed moral legitimacy of the practice of killing and consuming animals such as turkeys. I went so far in the piece as to argue that consuming turkeys and other animals raised in 'free range' circumstances is in effect no better morally than consuming animals raised in traditional intensive factory farming circumstances - for, after all, in both cases we kill sentient creatures with lives that are meaningful to them just as our lives are meaningful to us, even if non-human animals such as turkeys cannot, as we humans can, reflect on the ways in which their lives are meaningful to them.

I received a torrent of responses to my opinion piece, most of which were from people who were supportive of my views. A small but extremely vehement proportion of the responses was from individuals who excoriated me for my views, typically on the grounds that because many animals kill and eat animals, and because human beings are animals, it is therefore perfectly acceptable for human beings to eat non-human animals. Eating meat is 'natural', hence we are closer to nature by engaging in the slaughter and consumption of animals than we would be if we sought to avoid the consumption of animal flesh. From this standpoint, being an ethical vegetarian or vegan is the stuff of pure fancy, the expression of an utterly unrealistic desire to remake nature along the lines of a Disney movie - but one in which Bambi's mother does not get shot. Rather than engage in this sort of childish fantasy, these critics urged, let us celebrate our superiority over the rest of nature, let us take what belongs to us as a matter of natural right.

Given a 2500-year history of thinking in our culture according to which human beings are superior to non-human animals in virtue of possessing logos, the capacity for reason and language, what was most surprising to me in the responses I received to my opinion piece was not the vehemence of the criticism, but the fact that such a small proportion of those responding to my piece sought 
to defend the practice of consuming animal products. The history of Western philosophy is as much an articulation and reinforcement of human superiority and a justification of the consumption of animal products as it is anything else. From Aristotle's categorical exclusion of animals from the polis to the Stoics' denial that animals have any share in justice to Descartes's characterization of animals as biological machines to Kant's insistence that we have no direct moral duties to animals, the history of Western philosophy has generated and reinforced an ideology of human superiority and a conception of animals as instrumentalities for the satisfaction of human needs and desires.

It is for the sake of the 53 billion land animals who are killed annually for human consumption, not to mention the countless sea creatures, that this legacy of speciesism stands in dire need of critical examination. This number is provided by the United Nations World Agriculture Organization. For if it is permissible to eat animals, and if human beings are animals, why do we not eat our fellow human beings? I believe there are very clear reasons for this that ought to hold with equal force for non-human animals. Historically it has been argued that the human possession of logos constitutes a difference in kind between human and non-human animals, such that there are good reasons not to kill and eat human beings but equally good reasons why killing and eating non-human animals is perfectly permissible. Thinkers including Jeremy Bentham and Peter Singer have argued that because animals lack the capacity for rational reflection, they are incapable of grasping the remote past and distant future, and hence are incapable of contemplating what they have to lose by dying. Death, in other words, is not a harm for animals: animals cannot be said to possess an interest in what they cannot contemplate rationally, hence we do no wrong by depriving them of what they cannot contemplate in the abstract.

What convenient reasoning, offered by precisely that being that appears to be alone among living beings in possessing the capacity for rational contemplation. What few have bothered to ask is why we should suppose that a being can be harmed only by those things that it can grasp conceptually. The urgency of this question has been systematically obscured by a history of speciesistic thinking that has provided an easy justification for the use of animals as tools for the satisfaction of human desires. What has also been systematically obscured is a fundamental fact of existence that has largely been forgotten ever since Plutarch and Porphyry urged it on us: that animals are our kin, that they strive to realize their natural potential just as we seek to realize our own, and that we owe duties of justice to animals just as we owe duties of justice to our fellow human beings. First among these duties is the obligation to refrain from using animals or consuming animal products, and to let animal beings be in a manner that corresponds to their dignity as sentient beings.

Gary Steiner is John Howard Harris Professor of Philosophy at Bucknell University in Lewisburg, PA, USA. 